Einführung zum Thema

Onkologe $2021 \cdot 27: 524$

https://doi.org/10.1007/s00761-021-00953-4

Angenommen: 9. April 2021

(c) Springer Medizin Verlag $\mathrm{GmbH}$, ein Teil von Springer Nature 2021

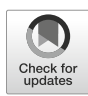

\author{
R. Gutzmer $\cdot$ C. Rose ${ }^{2} \cdot$ D. Vordermark 3 H. Schmidberger ${ }^{4}$ \\ 'Universitätsklinik für Dermatologie, Venerologie, Allergologie und Phlebologie, Johannes Wesling \\ Klinikum, Mühlenkreiskliniken Minden, Universitätsklinikum, Ruhr-Universität Bochum, Minden, \\ Deutschland \\ ${ }^{2}$ Dermatopathologie Lübeck, Lübeck, Deutschland \\ ${ }^{3}$ Universitätsklinik für Strahlentherapie, Universitätsklinikum Halle/Saale, Halle (Saale), Deutschland \\ ${ }^{4}$ Unimedizin Mainz, Mainz, Deutschland
}

\title{
Aktueller Stand bei nichtmelanozytären Hauttumoren
}

die inoperable Situation geführt. Dazu gehören molekular zielgerichtete Therapieansätze wie der Multikinaseinhibitor Imatinib beim Dermatofibrosarcoma protuberans (siehe Beitrag von Ugurel et al.) und die Hedgehog-Inhibitoren Sonidegib und Vismodegib beim Basalzellkarzinom (siehe Beitrag von Alter et al.). Immunologisch haben auch bei den nichtmelanozytären Hauttumoren die PD-1/PD-L1-Inhibitoren Einzug gehalten, so das Avelumab beim MerkelZell-Karzinom (siehe Beitrag Gutzmer et al.) und Cemiplimab beim kutanen Plattenepithelkarzinom (siehe Beitrag Leiter et al.).

Und die Entwicklung geht weiter: Da nicht alle Patienten von den medikamentösen Optionen in den inoperabel metastasierten Stadien profitieren, wird aktuell der Einsatz von Kombinationen im Rahmen von Studien untersucht, wie die Kombination von Hedgehog-Inhibitoren und PD-1-Inhibitoren beim Basalzellkarzinom oder die Kombination von Cetuximab und Avelumab beim kutanen Plattenepithelkarzinom.

Gleichzeitig wird der Einsatz der erfolgreichen Therapien in früheren Stadien untersucht, sprich in der adjuvanten Situation. Dazu gehören Studien mit Nivolumab (versus Beobachtung) beim Merkel-Zell-Karzinom sowie Cemiplimab und Pembrolizumab (versus Placebo) beim kutanen Plattenepithelkarzinom. Auch für Patienten mit nichtmelanozytären Hauttumoren, bei denen die chirurgische Entfernung nicht möglich ist oder ein hohes Rezidivrisiko besteht, ist also die Behandlung im Rahmen klinischer Studien eine Option. Dadurch wird sich auch in der Zukunft die Evidenzlage erweitern und das Spektrum der Therapieoptionen für unsere Patienten verbessern.

Die Beiträge in diesem Sonderheft des Onkologen zeigen diese faszinierenden Entwicklungen bei nichtmelanozytären Hauttumoren auf. Viel Spaß bei der Lektüre wünschen

Für die Schriftleiter

Ralf Gutzmer

Für die Herausgeber Heinz Schmidberger

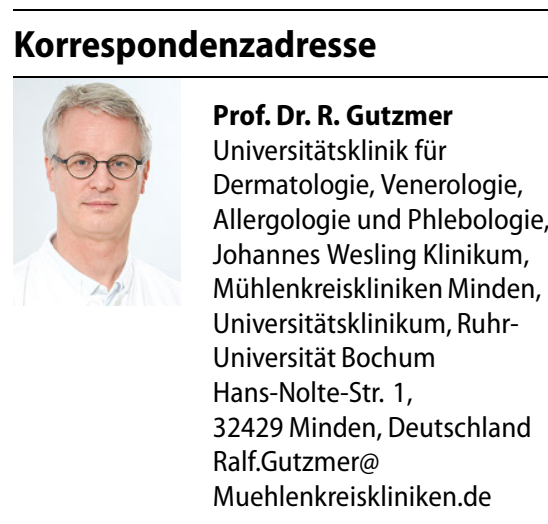

Interessenkonflikt. R. Gutzmer, C. Rose, D. Vordermark und H. Schmidberger geben an, dass kein Interessenkonflikt besteht. 